\title{
GENERALIZED FUNCTIONS AND ORTHOGONAL POLYNOMIALS ON THE UNIT CIRCLE
}

\author{
AHMED I. ZAYED
}

\begin{abstract}
ABSTRACr. In a recent paper, R. Morton and A. Krall introduced a new notion of orthogonality of polynomials on the real line in which a sequence of Chebyshev-type polynomials was shown to be orthogonal with respect to a moment generating linear functional. This linear functional is, in fact, a Schwartz distribution. If the polynomials are orthogonal in the classical sense, then the classical weight function can be recovered from the distributional one by means of the Fourier transform.

The purpose of this paper is to study the analogue of their results for polynomials orthogonal on the unit circle.
\end{abstract}

1. Introduction. In the last decade, the concept of orthogonality of polynomials has been generalized in a number of different ways [6,8]. Law and Sledd [6] used the three-term recurrence relation to define orthogonality in a way independent of a weight function.

Recently, R. D. Morton and A. M. Krall [8] introduced a new notion of orthogonality in which a family of polynomials $\left\{p_{n}(x)\right\}$ of the real variable $x$ was shown to be orthogonal with respect to a generalized function $w$ (Schwartz distribution) in the sense $\left\langle w, p_{n}(x) p_{m}(x)\right\rangle=\delta_{n m}$.

In fact, they showed that for any given sequence of real numbers $\left\{\mu_{i}\right\}_{i=0}^{\infty}$ satisfying the conditions:

(i) $\Delta_{k}=\operatorname{det}\left|\mu_{i+j}\right|_{i, j=0}^{k} \neq 0, k=0,1,2, \ldots$,

(ii) $\left|\mu_{k}\right|<c M^{k} k$ ! where $c, M$ are constants and $k=0,1,2, \ldots$, the Chebyshev polynomials $\left\{p_{n}(x)\right\}_{n=0}^{\infty}$ defined by $p_{0}(x)=1$ and

$$
p_{n}(x)=\left(\frac{1}{\Delta_{n-1}}\right)\left|\begin{array}{cccc}
\mu_{0} & \mu_{1} & \cdots & \mu_{n} \\
\vdots & \vdots & & \\
\mu_{n-1} & \mu_{n} & \cdots & \mu_{2 n-1} \\
1 & x & & x^{n}
\end{array}\right|, \quad n=1,2, \ldots,
$$

are orthogonal with respect to the linear functional

$$
w(x)=\sum_{n=0}^{\infty}(-1)^{n} \mu_{n} \frac{\delta^{(n)}(x)}{n !},
$$

Received by the editors April 20, 1982. Presented at the winter meeting of the Canadian Mathematical Society, Victoria, British Columbia, December 1981.

1980 Mathematics Subject Classification. Primary 42C05; Secondary 46F15.

Key words and phrases. Generalized functions, orthogonal polynomials.

(C)1983 American Mathematical Society 0002-9939/82/0000-1103/\$02.75 
where $\delta^{(n)}(x)$ denotes the $n$th derivative of the Dirac delta function. We call $w(x)$ a weight functional or a generalized weight function.

When the moments $\left\{\mu_{i}\right\}$ are those associated with the various classical orthogonal polynomials (Jacobi, Laguerre, Hermite), the classical weight function $w_{c}(x)$ can be recovered from the generalized (distributional) one $w(x)$ by taking the Fourier transform of the anayltic continuation of the inverse Fourier transform of $w(x)$.

It was rather a surprise to the authors that the Bessel polynomials, which are orthogonal on the unit circle in the sense

$$
\left\langle p_{n}(z), p_{m}(z)\right\rangle=\frac{1}{2 \pi i} \int_{|z|=1} p_{n}(z) p_{m}(z) e^{-2 / z} d z=\frac{(-1)^{n+1} 2}{2 n+1} \delta_{n, m},
$$

failed to yield completely to their technique.

An extension of the techniques used in [8] was motivated by the fact that they did not yield satisfying results when applied to the Bessel polynomials.

Indeed, by using the analytic representation

$$
\hat{w}(z)=\frac{1}{2 \pi i}\left\langle w(x), \frac{1}{x-z}\right\rangle ; \quad z \notin \operatorname{supp} w(x),
$$

of the weight functional $w(x)$ instead of its Fourier transform, Krall [5] was able to recover the classical weight function for the Bessel polynomials from the generalized one. In fact, his technique works for any polynomials $\left\{p_{n}(z)\right\}$ orthogonal (in the classical sense) on any closed rectifiable Jordan curve $\gamma$ provided that orthogonality is defined by means of the inner product

$$
(f, g)=\int_{\gamma} f(z) g(z) w_{c}(z) d z
$$

where the weight function $w_{c}(z)$ has an essential singularity at some point inside $\gamma$. This follows easily from the arguments given in [2, p. 119] and [5].

However, if one defines orthogonality by means of the inner product

$$
(f, g)=\int_{\gamma} f(z) \overline{g(z)} w_{c}(z) d z,
$$

Krall's technique is no longer applicable.

We denote the classes of polynomials orthogonal on the unit circle in the sense of (1.1) and (1.2) by $Q$ and $\Re$, respectively.

The purpose of this article is to extend the results of [8] and [5] to the class $\mathscr{B}$. Our techniques differ from theirs for the following reasons:

(i) For the class $Q$, it is known that the weight functions $w_{c}(z)$ must have singular points inside the unit circle. On the contrary, the weight functions for the class $\mathscr{B}$ are the boundary values of harmonic functions in the unit disc [3, p. 17].

(ii) As a consequence of (i), it becomes evident that the theory of Schwartz distributions is no longer able to provide a natural setting for the generalized weight functions. For, the boundary values of harmonic functions inside the disc are not necessarily Schwartz distributions on the unit circle. 
2. Preliminaries. Let $\mathcal{H}$ denote the linear space of analytic complex-valued functions on the unit circle $T$. In terms of Fourier series developments, the class $\mathcal{H}$ may be defined as

$$
\mathscr{H}=\left\{\phi: \phi(\theta)=\sum_{-\infty}^{\infty} c_{k} e^{i k \theta}, \limsup _{|k| \rightarrow \infty}\left|c_{k}\right|^{1 /|k|}<1\right\} .
$$

A locally convex topology is defined on $\mathcal{H}$ by means of the following base for the neighborhood system at the origin

$$
V(\alpha)=\left\{\phi \in \mathcal{H}:\left|c_{k}\right| \leqslant \alpha_{|k|} \text { for all } k\right\}
$$

where $\alpha=\left\{\alpha_{k}\right\}_{k=0}^{\infty}, \alpha_{k} \geqslant \alpha_{k+1}>0$ for all $k$, and $\lim _{k \rightarrow \infty}\left(\alpha_{k+1} / \alpha_{k}\right)=1$.

Provided with this topology, $\mathcal{H}$ is a Fréchet-Montel space that is not metrizable [4]. The strong dual $\mathfrak{H}^{*}$ of $\mathcal{H}$ is the space of hyperfunctions on $T$. $\mathfrak{H}^{*}$ is also a Fréchet-Montel space whose strong dual $\mathfrak{H}^{* *}$ is $\mathcal{H}^{*}$. The space $\mathcal{H}^{*}$ can be characterized in terms of Fourier series as follows: $f \in \mathcal{H}^{*}$ if and only if

$$
f=\sum_{-\infty}^{\infty} a_{n} e^{i n \theta}, \quad \limsup _{|n| \rightarrow \infty}\left|a_{n}\right|^{1 /|n|} \leqslant 1
$$

where $\left\langle f, e^{-i n \theta}\right\rangle=a_{n}$.

It is well known that $f$ is a Schwartz distribution on $T$ if and only if $\left|a_{n}\right| \leqslant$ $B(|n|+1)^{p}$ for some constants $B$ and $p$.

Let $\mathcal{F}$ denote the space of complex-valued harmonic functions in the unit disc $\mathscr{D}$ provided with the topology of uniform convergence on compact subsets of $\mathscr{D}$.

Provided with this topology, the space $\mathscr{F}$ is topologically and algebraically isomorphic to the space $\mathcal{H}^{*}[4]$.

In fact, if $f(r, \theta) \in \mathscr{F}$, then

$$
\lim _{r \rightarrow 1} f(r, \theta)=\tilde{f}(\theta) \in \mathcal{H}^{*}
$$

where the convergence, of course, is in the sense of $\mathcal{H}^{*}$.

Moreover, if $\tilde{f}(\theta) \in \mathcal{K}^{*}$, then

$$
P_{r}(t-\theta) * \tilde{f}(\theta)=f(r, t) \in \mathscr{F}
$$

where $P_{r}(t)$ is the Poisson kernel for the unit disc and * stands for the convolution.

We choose the duality $\langle f, \phi\rangle$ on $\mathcal{H}^{*} \times \mathcal{H}$ to be linear on $\mathcal{H}$ and skew linear on HC*

Hence, if $f=\sum_{-\infty}^{\infty} a_{n} w^{n} \in \mathcal{H}^{*}$ and $\phi=\sum_{-\infty}^{\infty} b_{n} w^{n} \in \mathcal{H}$ where $w=e^{i \theta}$, then it is readily seen that

$$
\langle f, \phi\rangle=\sum_{-\infty}^{\infty} \bar{a}_{n} b_{n}<\infty
$$


3. Orthogonality and generalized weight functions. Let $\left\{c_{n}\right\}_{-\infty}^{\infty}$ be a sequence of complex numbers subject to the conditions:

$$
\begin{aligned}
& \Delta_{k}=\operatorname{det}\left[c_{i-j}\right]_{0}^{k}=\left|\begin{array}{cccc}
c_{0} & c_{1} & \cdots & c_{k} \\
c_{-1} & c_{0} & \cdots & c_{k-1} \\
\vdots & \vdots & & \vdots \\
c_{-k} & c_{-k+1} & & c_{0}
\end{array}\right| \neq 0 ; \\
& c_{-k}=\bar{c}_{k}, k=0,1,2, \ldots, \\
& \limsup _{|n| \rightarrow \infty}\left|c_{n}\right|^{1 /|n|} \leqslant 1 .
\end{aligned}
$$

We call the numbers $\left\{c_{n}\right\}_{-\infty}^{\infty}$ generalized trigonometric moments. Consider the classical system of Chebyshev polynomials defined by $p_{0}(z)=1$ and for $k=1,2, \ldots$,

$$
p_{k}(z)=\frac{1}{\Delta_{k-1}}\left|\begin{array}{cccc}
c_{0} & c_{-1} & \cdots & c_{-k} \\
c_{1} & c_{0} & \cdots & c_{-k+1} \\
\vdots & \vdots & & \vdots \\
c_{k-1} & c_{k-2} & & c_{-1} \\
1 & z & & z^{k}
\end{array}\right|=z^{k}+\cdots .
$$

THEOREM 3.1. The polynomials $\left\{p_{k}(z)\right\}_{0}^{\infty}$ are orthogonal with respect to the hyperfunction $f(w)=\sum_{-\infty}^{\infty} c_{n} w^{n}$ in the following sense:

$$
\left\langle f, p_{k}(w) \overline{p_{n}(w)}\right\rangle=\left\{\begin{array}{l}
0 \quad \text { if } k \neq n, k, n=0,1,2, \ldots, \\
h_{k}=\Delta_{k} / \Delta_{k-1} \neq 0 \text { if } k=n .
\end{array}\right.
$$

Proof. It is sufficient to observe that

$$
\begin{aligned}
\left\langle f, p_{k}(w) w^{-m}\right\rangle & =\frac{1}{\Delta_{k-1}} \mid \begin{array}{cccc}
c_{0} & c_{-1} & \ldots & c_{-k} \\
c_{1} & c_{0} & \cdots & c_{-k+1} \\
\vdots & \vdots & & \vdots \\
c_{k-1} & c_{k-2} & c_{-1} \\
c_{m} & c_{m-1}
\end{array} \\
& =\left\{\begin{array}{l}
0 \text { if } 0 \leqslant m \leqslant k-1, \quad k=1,2, \ldots, \\
h_{k} \text { if } m=k ;
\end{array}\right. \\
& \left\langle f, p_{0}(w) \overline{p_{0}(w)}\right\rangle=c_{0} .
\end{aligned}
$$

Q.E.D.

Let $\left\{a_{k}\right\}_{k=0}^{\infty}$ be a sequence of parameters defined by

$$
a_{k}=\left(1 / h_{k}\right)\left\langle f, \bar{w} \bar{p}_{k}(w)\right\rangle \text {. }
$$

Then one can easily show that

$$
p_{k+1}(z)=z p_{k}(z)-\bar{a}_{k} p_{k}^{*}(z), \quad p_{k}^{*}(z)=z^{k} \overline{p_{k}(z)}, \quad k=0,1,2, \ldots,
$$




$$
\begin{aligned}
& \bar{a}_{k} p_{k+2}(z)=\left(\bar{a}_{k} z+\bar{a}_{k+1}\right) p_{k+1}(z)-z \bar{a}_{k+1}\left(1-\left|a_{k}\right|^{2}\right) p_{k}(z), \\
& k=0,1,2, \ldots, p_{0}(z)=1, p_{1}(z)=z-\bar{a}_{0} .
\end{aligned}
$$

Furthermore, if we assume that $\left|a_{k}\right| \neq 1$ for all $k$, then the system of polynomials $\left\{p_{k}(w)\right\}_{0}^{\infty}$ satisfying (3.6) or (3.7) is orthogonal with respect to the hyperfunction $f=\lambda \sum_{k=-\infty}^{\infty} c_{k} w^{k}$, where $\lambda$ is an arbitrary constant.

The polynomials $\left\{q_{k}(z)\right\}_{0}^{\infty}$ of the second kind are defined by $q_{0}(z)=1$ and

$$
q_{k}(z)=\frac{1}{c_{0}}\left\langle f(w), \frac{w+z}{w-z}\left(p_{k}(w)-p_{k}(z)\right)\right\rangle, \quad k=1,2, \ldots
$$

The reader should be able to prove the following relations:

$$
\begin{gathered}
q_{k+1}(z)=z q_{k}(z)+\bar{a}_{k} q_{k}^{*}(z), \quad k=0,1,2, \ldots, \\
p_{k}^{*}(z) q_{k}(z)+p_{k}(z) q_{k}^{*}(z)=\left(2 h_{k} z^{k}\right) / c_{0}, \quad k=0,1,2, \ldots, \\
\frac{c_{0} q_{k}^{*}(z)}{2 p_{k}^{*}(z)}=\frac{c_{0}}{2}+c_{1} z+\cdots+c_{k} z^{k}+O\left(z^{k+1}\right), \quad k=0,1,2, \ldots,
\end{gathered}
$$

in a sufficiently small neighborhood of $z=0$.

Finally, define the analytic function $F(z)$ by

$$
F(z)=\frac{1}{2 \pi c_{0}}\left\langle f(w), \frac{w+z}{w-z}\right\rangle, \quad \text { for } z \notin T .
$$

It is known [3, Theorem 13.3] that the condition $\left\{\Delta_{k}>0\right\}_{0}^{\infty}$ is both necessary and sufficent for the existence of a bounded nondecreasing function $\sigma(\theta)$ on $[0,2 \pi]$ such that

$$
c_{k}=\frac{1}{2 \pi} \int_{0}^{2 \pi} e^{-i k \theta} d \sigma(\theta), \quad k=0,1,2, \ldots
$$

Consequently, the analytic function $F(z)$ defined by

$$
F(z)=\left(2 / c_{0}\right)\left(c_{0} / 2+c_{1} z+c_{2} z^{2}+\cdots\right), \quad|z|<1,
$$

can be written in the form

$$
F(z)=\frac{1}{2 \pi c_{0}} \int_{0}^{2 \pi} \frac{w+z}{w-z} d \sigma(\theta),
$$

where $w=e^{i \theta}$.

It is easy to see that $F(z)$ is pseudopositive, i.e., has a positive real part, hence it belongs to the Hardy space $H^{p}$ for all $0<p<1$. Therefore, it follows that $F(z)$ has a finite nontangential limit almost everywhere on the unit circle [11]. We denote this limit by $F\left(e^{i \theta}\right)$ and set $f\left(e^{i \theta}\right)=\operatorname{Re} F\left(e^{i \theta}\right)$.

The function $\sigma(\theta)$ can be recovered from $F(z)$ by means of the inversion formula (cf. [3, Formula 11.7])

$$
\frac{\sigma(\theta+0)+\sigma(\theta-0)}{2}=\text { constant }+c_{0} \lim _{r \rightarrow 1^{-}} \int_{0}^{\theta} \operatorname{Re} F\left(r e^{i \phi}\right) d \phi .
$$


Moreover,

$$
\lim _{r \rightarrow 1} \operatorname{Re} F\left(r e^{i \theta}\right)=D \sigma(\theta)
$$

whenever the symmetric derivative

$$
D \sigma(\theta)=\lim _{h \rightarrow 0^{-}} \frac{\sigma(\theta+h)-\sigma(\theta-h)}{2 h}
$$

exists (cf. [11, p. 99]). In particular, if $d \sigma(\theta)=\sigma^{\prime}(\theta) d \theta+d \mu(\theta)$ where $\mu$ is a singular measure, then

$$
\lim _{r \rightarrow 1} \operatorname{Re} F\left(r e^{i \theta}\right)=\sigma^{\prime}(\theta)=f\left(e^{i \theta}\right) \quad \text { a.e. }
$$

Furthermore, if we assume that

$$
\lim _{n \rightarrow \infty}\left(\Delta_{n} / \Delta_{n-1}\right)
$$

exists, then

$$
\lim _{r \rightarrow 1} \lim _{n \rightarrow x} \frac{h_{n}}{\left|p_{n}^{*}\left(r e^{i \theta}\right)\right|^{2}}=\sigma^{\prime}(\theta) \text { a.e. in }[0,2 \pi] .
$$

See, for example, Theorem 21.1 and Formula 26.22 in [3].

The purpose of this section is to study the analogue of the preceding results when the condition $\left\{\Delta_{k}>0\right\}$ is relaxed to $\left\{\Delta_{k} \neq 0\right\}$.

THEOREM 3.2 Let $F(z)=\sum_{k=0}^{\infty} c_{k} z^{k}$ be a holomorphic function in ( 12 . Then the condition $\left\{\Delta_{k} \neq 0\right\}$ is necessary and sufficient for the existence of a sequence of polynomials $\left\{p_{k}(z)\right\}_{0}^{\infty}$ orthogonal on $T$ with respect to

$$
\lim _{z \rightarrow w} \operatorname{Re} F(z)=f(w) .
$$

Proof. Sufficiency. Define the functional $f$ by

$$
c_{-k}=\left\langle f, w^{k}\right\rangle ; \quad c_{-k}=\bar{c}_{k}, \quad k=0, \pm 1, \pm 2, \ldots
$$

Since $F(z)$ is holomorphic in $\left.{ }^{1}\right)$, then $\lim \sup _{n \rightarrow \infty}\left|c_{n}\right|^{1 / n} \leqslant 1$. Construct the polynomials $\left\{p_{k}(z)\right\}_{k=0}^{\infty}$ as in (3.3), hence by Theorem 3.1 they are orthogonal with respect to the hyperfunction $f(w)=\sum_{-\infty}^{\infty} c_{k} w^{k}$. But from the uniqueness of the representation of hyperfunctions, it follows that

$$
\lim _{z \rightarrow w} \operatorname{Re} F(z)=f(w) .
$$

Necessity. Let

$$
f(w)=\lim _{z \rightarrow w} \operatorname{Re} F(z)=\sum_{-\infty}^{\infty} c_{k} w^{k} \quad \text { and } \quad\left\langle f, p_{k}(w) \overline{p_{n}(w)}\right\rangle=h_{k} \delta_{k, n} .
$$

Let $p_{k}(z)=\sum_{i=0}^{k} a_{i}^{k} z^{i}$; therefore we have

$$
\left\langle f, p_{k}(w) w^{-n}\right\rangle=\sum_{i=0}^{k} a_{i}^{k} c_{n-i}=0 \text { for } 0 \leqslant n \leqslant k-1, k=1,2, \ldots,
$$


and

$$
\left\langle f, p_{k}(w) w^{-k}\right\rangle=\sum_{i=0}^{k} a_{i}^{k} c_{k-i}=\frac{h_{k}}{\bar{a}_{k}^{k}} \neq 0 .
$$

These are $k+1$ nonhomogeneous equations in $k+1$ unknowns, hence they have a unique solution if and only if $\Delta_{k} \neq 0$. Q.E.D.

(3.19) is an analogue of (3.16). Nevertheless, since the convergence in (3.16) is taken in the pointwise (local) sense while that in (3.19) is taken in the hyperfunction (global) sense, one immediately realizes that the latter is not indeed a true generalization of the former.

To obtain a local version of (3.19), we introduce Johnson's characterization of hyperfunctions.

$f$ is a hyperfunction on $T$ if and only if there is a sequence $\left\{f_{n}\right\}_{0}^{\infty}$ of continuous functions on $T$ satisfying

$$
\lim _{n \rightarrow \infty}\left(n !\left\|f_{n}\right\|_{\infty}\right)^{1 / n}=0
$$

such that

$$
f=\sum_{n=0}^{\infty} f_{n}^{(n)} \quad \text { where } f_{n}^{(n)}=\frac{d^{n}}{d \theta^{n}} f_{n}(\theta), 0 \leqslant \theta \leqslant 2 \pi .
$$

Definition. The hyperfunction $f$ on $T$ is said to have a value $\gamma$ at $\theta_{0}$ if there exist a representation $\sum_{n=0}^{\infty} f_{n}^{(n)}$ of $f$ satisfying (3.20), a sequence of polynomials $\left\{g_{n}\right\}$ with degree of $g_{n}$ smaller than $n$ and a sequence of complex numbers $\left\{\gamma_{n}\right\}$ such that for each $\varepsilon>0$, there exists a $\delta>0$ such that

$$
\left|\frac{f_{n}(\theta)-g_{n}(\theta)}{\left(\theta-\theta_{0}\right)^{n}}-\frac{\gamma_{n}}{n !}\right| \leqslant \frac{\varepsilon^{n+1}}{n !} \text { for } 0<\left|\theta-\theta_{0}\right|<\delta,
$$

$n=0,1,2, \ldots$, and $f_{0}\left(\theta_{0}\right)=\gamma_{0}$ where $\gamma=\sum_{n=0}^{\infty} \gamma_{n}$.

(3.21) is equivalent to saying that $f_{n}$ has $n$th order Peano derivative, namely $\gamma_{n}$, at the point $\theta_{0}$.

In particular, if $f$ is a continuous function at $\theta_{0}$, then its value at $\theta_{0}$ as a hyperfunction is $f\left(\theta_{0}\right)$. Moreover, if $f$ is a locally integrable function and $\sigma(\theta)=$ $\int_{0}^{\theta} f(t) d t$ is differentiable at $\theta_{0}$, then the value of the hyperfunction $f$ at $\theta_{0}$ is $\sigma\left(\theta_{0}\right)$.

For more details see $[\mathbf{7 , 9}, \mathbf{1 0}]$.

The following theorem generalizes (3.16).

THEOREM 3.3. Let $F(z)$ and $f(w)$ be given as in Theorem 3.2. In addition, let $f$ have a value at $w_{0}$ and denote this value by $f\left(w_{0}\right)$. Then,

$$
\lim _{z \rightarrow w_{0}} \operatorname{Re} F(z)=f\left(w_{0}\right) \text {. }
$$

Proof. The same as in the main theorem of [9].

The next theorem is a generalization of (3.18). 
THEOREM 3.5. Let $\left\{p_{k}(z)\right\},\left\{h_{k}\right\}$ and $f(w)$ be given as in Theorem 3.1. Then

$$
\lim _{k \rightarrow \infty} \frac{h_{k}}{\left|p_{k}^{*}(w)\right|^{2}}=f(w)
$$

where the convergence is understood to be in the sense of hyperfunctions.

Proof. From (3.10) we obtain

$$
\overline{p_{k}^{*}(z)} q_{k}^{*}(z)+p_{k}^{*}(z) \overline{q_{k}^{*}(z)}=2 h_{k} / c_{0},
$$

and since $p_{k}^{*}(0) \neq 0$, it easily follows from (3.11) that

$$
\frac{c_{0}}{2}\left(\frac{q_{k}^{*}(z)}{p_{k}^{*}(z)}+\frac{\overline{q_{k}^{*}(z)}}{\overline{p_{k}^{*}(z)}}\right)=c_{0} \operatorname{Re} \frac{q_{k}^{*}(z)}{p_{k}^{*}(z)}=\frac{h_{k}}{\left|p_{k}^{*}(z)\right|^{2}}=\sum_{m=-\infty}^{\infty} a_{m} r^{|m|} e^{i m \theta}
$$

where $0 \leqslant r<\delta_{k}$ for some $\delta_{k}$ and $a_{m}=c_{m}$ for $m=-k,-k+1, \ldots, 0, \ldots, k$.

Let us denote those zeros of $p_{k}^{*}(z)$ that lie inside and on the unit circle by $z_{1}, \ldots, z_{j}$ and their corresponding multiplicity by $\alpha_{1}, \ldots, \alpha_{j}$ where $1 \leqslant j \leqslant k$. Without loss of generality, we may assume that no two zeros lie on the same circle with the origin as a center. Arrange these zeros so that $0<\delta_{k}<\left|z_{1}\right|<\left|z_{2}\right|<\cdots<\left|z_{j}\right| \leqslant 1$.

Let $z_{1}=r_{1} w_{1}$; then by the same argument given in [1], one can show that

$$
\lim _{r \rightarrow r_{1}^{-}} \frac{1}{\left(r w-r_{1} w_{1}\right)^{\alpha_{1}}}=\frac{1}{\left(r_{1} w-r_{1} w_{1}\right)^{\alpha_{1}}}-\frac{i \pi \delta^{(n)}\left(r_{1} w-r_{1} w_{1}\right)}{\alpha_{1} !}
$$

where the limit is taken in the sense of generalized functions and the terms on the right side are defined as those in [1, p. 33].

Now one can easily see that $\lim _{r \rightarrow 1}\left(1 / p_{k}^{*}(z)\right)$ is in general a singular generalized function on $T$ with at most $k$ singular points. The same argument applies to $\lim _{r \rightarrow 1}\left(h_{k}:\left|p_{k}^{*}(z)\right|^{2}\right)$.

Define $f_{k}(w)$ by

$$
f_{k}(w)=\lim _{r \rightarrow 1^{-}} \frac{h_{k}}{\left|p_{k}^{*}(z)\right|^{2}} .
$$

From (3.26), we obtain that

$$
f_{k}(w)=\sum_{m=-\infty}^{\infty} a_{m} w^{m}, \quad a_{m}=c_{m} \text { for } m=-k, \ldots, 0, \ldots, k .
$$

Now if $f_{k}(w)=\sum_{m=-\infty}^{\infty} a_{m}^{k} w^{m}$ is a sequence of generalized functions such that $f_{k} \rightarrow f=\sum_{m=-\infty}^{\infty} a_{m} w^{m}$, then $\lim _{k \rightarrow \infty} a_{m}^{k}=a_{m}$ for all $m$. This follows from the fact that $f_{k} \rightarrow f$ implies $\left(f_{k}, w^{n}\right) \rightarrow\left(f, w^{n}\right)$ for all $n$.

Combining all these facts finally yields

$$
\lim _{k \rightarrow \infty} \lim _{r \rightarrow 1} \frac{h_{k}}{\left|p_{k}^{*}(z)\right|^{2}}=\lim _{k \rightarrow \infty} \frac{h_{k}}{\left|p_{k}^{*}(w)\right|^{2}}=f(w)=\sum_{m=-\infty}^{\infty} c_{m} w^{m} . \quad \text { Q.E.D. }
$$




\section{REFERENCES}

1. J. M. Gel'fand and G. E. Shilov, Generalized functions, vol. 1, Academic Press, New York, 1964.

2. Ya. L. Geronimus, Orthogonal polynomials, Amer. Math. Soc. Transl. (2) 108 (1977), 37-130.

3. , Polynomials orthogonal on a circle and their applications, Amer. Math. Soc. Transl. (1) 3 (1968), 1-78.

4. G. Johnson, Harmonic functions on the unit disk. I, Illinois J. Math. 12 (1968), 366-385.

5. A. M. Krall, Orthogonal polynomials through moment generating functionals, SIAM J. Math. Anal. 9 (1978), 600-603.

6. A. G. Law and M. B. Sledd, Normalizing orthogonal polynomials by using their recurrent relation, Proc. Amer. Math. Soc. 48 (1975), 505-507.

7. S. Lojasiewicz, Sur la valeur et la limite d'une distribution dans un point, Studia Math. 16 (1957), $1-36$.

8. R. D. Morton and A. M. Krall, Distributional weight functions for orthogonal polynomials, SIAM J. Math. Anal. 9 (1978), 604-626.

9. G. Walter, Local boundary behavior of harmonic functions, Illinois J. Math. 12 (1968), 366-385.

10. A. Zayed and G. Walter, Series of orthogonal polynomials as hyperfunctions, SIAM J. Math. Anal. 13 (1982), 664-675.

11. A. Zygmund, Trigonometric series, Cambridge Univ. Press, London and New York, 1968.

Department of Mathematics, California Polytechnic State University, San luis Obispo, CALIFORNIA 93401 